www.jmscr.igmpublication.org Impact Factor 5.244

Index Copernicus Value: 83.27

ISSN (e)-2347-176x ISSN (p) 2455-0450

crossref DOI: _https://dx.doi.org/10.18535/jmscr/v4i12.94

Journal Of Medical Science And Clinical Research

\title{
Validation of Thorax Trauma Severity Score Using Early Emergency Department Computed Tomography (MDCT 64 Slice/ Multirow Detector CT)
}

\author{
Authors \\ Anurag Rai, Parijat Suryavanshi, Suresh Kumar, Umesh Kumar \\ Department of Surgery, King George's Medical University, Lucknow
}

\begin{abstract}
Background: Rapid and accurate assessment of the thoracic trauma is important to direct life-saving and definitive management. In traumatic lung injury patients, till date thorax trauma severity score (TTSS) was calculated by using conventational X raychest. MDCT 64 slice (Multirow Detector -CT) Scan has significantly shorter scanning time and higher injury detection rate than conventional X-ray chest and single slice CT Scan (SSCT). We observed role of early emergency CT scan Thorax (MDCT) in traumatic lung injury patients. Objective: The aim of this study is to validate thorax trauma severity score (TTSS) using early MDCT 64 slice (Multirow Detector -CT).

Material \& Methods: In this retrospective study, 80 patients of Isolated chest injuries (blunt and penetrating chest injuries) having AIS THORAX > 1 admitted at King George's Medical University Trauma Centre, Luck now ,between June 2012 to July 2013 who were scanned early in Emergency Department with MDCT 64-slice and helical single slice CT using the standardized multiple trauma protocol(ATLS guidelines), were eligible for the study.All records were noted from case sheet from the time of admission and subsequently followed regarding suitable management like surgical intervention or mechanical ventilation or thorax related complication or follow up complications.

Results: Of the 80 patients included in the study, 52 (65\%) developed thoraxrelated complications. The overall inhospital mortality rate was $10 \%$. The receiver operating characteristic(ROC) curve for predicting mortality demonstrated an adequate discrimination by a statistically significant higher Area under curve (AUC) in patients who died of thorax-related complications than in patients who survived ( $P=0.002$, confidence interval [CI] 95\% for TTSS $M D C T)$. In patients who developed ARDS the TTSS was significant higher $(P=0.0001, C I 95 \%)$. Area under curve (AUC) of TTSS ROC curve was highest for MDCT (0.81) then for Single slice CT (0.79) and least for x-ray(0.78), indicating highest sensitivity, specificity and predictive ability of MDCT, then SSCT and conventional $x$-ray for predicting mortality in emergency department. Area under curve (AUC) of TTSS ROC curve was highest for MDCT( 0.92) then for Single slice CT ( 0.85) and least for x-ray (0.81), indicating highest sensitivity, specificity and predictive ability of MDCT, then Single slice CT and conventional x-ray for predicting ARDS.

Conclusion: This study validate the Thoracic Trauma Severity Score(TTSS) with new emerging concept of early emergency Department Muti- row detector CT in secondary survey for predicting mortality in critically ill thoracic injury patients. TTSS MDCT (thorax trauma severity score on the basis of MDCT64 slice findings) appears capable of predicting ARDS more precisely then TTSSx-ray (thorax trauma severity score on the bases of chest x-ray findings).Thus shows the significance of early emergency MDCT in diagnostics and treatment decisions in traumatic lung injured patients.
\end{abstract}

Keywords - Multi-detector computed tomography, Thorax trauma severity score, acute respiratory distress syndrome, Emergency department CT, and Receiver operating characteristic.abberiveated injury score (AIS). 


\section{INTRODUCTION}

Blunt chest trauma is one of the most common problems experienced in road traffic accidents. Death due to chest trauma composes half of all trauma deaths and usually occurs immediately after the chest injury ${ }^{[1]}$. Rapid and accurate assessment of the chest in blunt trauma patients is important to direct life-saving and definitive management. Solid risk stratification of thoracic trauma is needed for individual management of ventilation, intensive care support, and surgical strategy selection to reduce morbidity and mortality ${ }^{[2,3]}$. A more rapid diagnostic workup may be achieved when using whole-body $\mathrm{CT}$ as a primary diagnostic tool compared with conventional use of radiography, combined with abdominal ultrasound and organ-focused CT ${ }^{[4]}$. Furthermore, the single-pass whole-body CT protocols may result in significantly shorter scanning time than the segmental conventional protocol ${ }^{[5]}$. The initial experience with the most advanced technique of a 64-row MDCT scanner indicates further time savings, especially in the reformatting and evaluation interval ${ }^{[6]}$. Compared with single-row-detector systems, MDCT scanners allow faster data collection and thinner slices that support more demanding clinical applications and present new research opportunities. Injury Severity Scoring is a process by which complex and variable patient data is reduced to a single number. A scoring system that can help predict thorax related complications in thoracic trauma patients is needed. For this in 2000 Pape et al ${ }^{[7]}$ developed the Thorax Trauma Severity Score (TTSS). As Manuscript (excluding authors' names and affiliations) Table 1, the TTSS combines the patient's age, resuscitation parameters, and radiological assessment of the thorax. After the first publication in 2000 the score has never been validated by any other independent study or by any other advanced radiological tool like CT (as described by Pape, Thorax Trauma Severity Score uses the radiological findings of $x$-ray chest ${ }^{[7]}$ Tab 1 . The association of the score with thoracic related death and mortality has not been explored. The aim of the present study was to evaluate and to validate the Thorax Trauma Severity Score (TTSS) on the bases of radiological findings of an early emergency department Computed Tomography (MDCT 64 slice- Multirow detector computed tomography) for predicting mortality and ARDS.

\section{MATERIALS AND METHODS}

In this retrospective study, patients of Isolated chest injuries (blunt and penetrating chest injuries) having AISTHORAX > 1(abbreviated injury score for thorax) admitted at King George's Medical University Trauma Centre, Luck now ,between June 2012 to July 2013 who were scanned early in Emergency Department with MDCT 64-slice and helical single slice CT. In our centre, chest trauma patient managed according to standardized multiple trauma protocol in ATLS (advanced trauma life support) .In Emergency Department MDCT 64-slice and helical single slice CT, both were done at the time of secondary survey on second and third day. All records were noted from case sheet from the time of admission and subsequently followed regarding suitable management like surgical intervention or mechanical ventilation or thorax related complication or follow up complications. Written informed consent and ethical committee approval was not required because of the retrospective nature of the investigation. We excluded patients of infective traumatic lung injuries, polytruma and Infected parenchymal lung diseases. Selection of patient was random on the day of admission. Medical records were reviewed for age, gender, and mechanism of injury, intensive care unit stay, and resuscitation length, thorax-related Complications like pneumonia, pneumothorax, persistent hematothorax, ARDS and mortality. MDCT findings of case were retrieved from radiological reports. These findings were compared with single slice CT radiological findings and digital chest $\mathrm{x}$ ray findings, follow-up studies, operative findings and autopsy findings in death reported cases. The TTSS calculated by all 3 available radiological 
tools (MDCT 64 slice, single slice helical CT and $\mathrm{X}$-ray chest) employing 5 specific parameters: rib fractures, lung contusion, $\mathrm{PaO} 2 / \mathrm{FiO} 2$ ratio, age, and pleural involvement. Injury reported in both CT was compared with references like other diagnostic tools or operative findings or autopsy findings to know about missed injuries. The data in the present study were analyzed using crosstabulation. Summary measurements were presented as means with standard deviation (SD) or as medians with 25 th and 75th percentiles. Categorical data were analyzed using the Chisquare test or Fisher's exact test. . The analyses were conducted using SPSS (version 16.0, SPSS inc., Chicago, Ill.). The association between various parameters was evaluated using univarieat analysis. Predictive values were calculated using a receiver operating characteristic curve (ROC) analysis. Statistical significance was defined as $\mathrm{P}$ $<0.05$.

\section{RESULTS}

Of the total 110 patients presented in Emergency Department(ED) and registered in the study period with an injury to the chest, 30 with minor chest trauma did not score any point on the AISthorax were excluded. So, 80 patients were included in the study. Demographic data are presented in Table 2.Of the 80 patients included in the study, $52(65 \%)$ developed thorax-related complications. The overall in-hospital mortality rate was $10 \%$. Mortality was correlated with high TTSS. The receiver operating characteristic curve (ROC) was used to demonstrate the sensitivity and specificity of the TTSS for predicting mortality during hospital stay. The receiver operating characteristic (ROC) curve for predicting mortality demonstrated an adequate discrimination by a statistically significant higher Area under curve (AUC) among the patients who died of thorax-related complications than in patients who survived $(\mathrm{P}$ $=0.002$, confidence interval $[\mathrm{CI}] 95 \%$ for TTSSMDCT). In patients who developed ARDS the TTSS was significant higher $(\mathrm{P}=0.0001, \mathrm{CI}$ 95\%).When TTSS evaluated with radiological findings of MDCT, single slice CT along with conventional $\mathrm{x}$-ray, the score was highest for MDCT in both group of patients(dead / alive and ARDS / non ARDS), with highly significant values as shown inTab3. TTSSMDCT > TTSSSSCT > TTSSX-RAY Area under curve (AUC) of TTSS ROC curve was highest for MDCT (0.81) $(95 \% \mathrm{CI}=0.70-0.93) \mathrm{p}=0.004$, then for Single slice CT (0.79) $(95 \% \mathrm{CI}=0.66-0.92)$ $\mathrm{p}=0.006$ and least for $\mathrm{x}$-ray $(0.78),(95 \% \mathrm{CI}=0.62$ 0.94) $\mathrm{p}=0.008$ indicating highest sensitivity, specificity and predictive ability of MDCT, then SSCT and conventional x-ray for predicting mortality in emergency department as shown in fig1. Area under curve (AUC) of TTSS ROC curve was highest for MDCT( 0.92) (0.86-0.97), $\mathrm{p}=0.001$ then for SSCT $(0.85)(0.77-0.93)$, $\mathrm{p}=0.001$ and least for $\mathrm{x}$-ray (0.81) $(0.72-0.90)$, $\mathrm{p}=0.001$, indicating highest sensitivity, specificity and predictive ability of MDCT, then SSCT and conventional $\mathrm{x}$-ray for predicting ARDS as shown in figure 2. 


\section{JMSCR Vol||04||Issue||12||Page 14887-14894||December}

Table-1: The TTSS developed by Pape et al ${ }^{[7]}$ to predict mortality in Thoracic Trauma patients; Point assignment corresponds to the Grade of the injury. The grades ranged from 0 (normal function) to 4 (most severe). Since the impact of Grade 4 injuries was higher, the grade and points were raised to $\mathrm{V}$ and 5 respectively. For calculation of the total score, all categories are summed. A minimum value of 0 points and a maximum value of 25 points can be achieved;

\begin{tabular}{|c|c|c|}
\hline Parameter & Finding & Points \\
\hline \multirow[t]{5}{*}{ Age } & $<30$ years of age & 0 \\
\hline & 30 to 41 years of age & 1 \\
\hline & 42 to 54 years of age & 2 \\
\hline & 55 to 70 years of age & 3 \\
\hline & $>70$ years of age & 5 \\
\hline \multirow[t]{5}{*}{$\mathrm{PaO}_{2}$ to $\mathrm{FIO}_{2}$ ratio } & $>400$ & 0 \\
\hline & $301-400$ & 1 \\
\hline & $201-300$ & 2 \\
\hline & $150-200$ & 3 \\
\hline & $<150$ & 5 \\
\hline \multirow[t]{6}{*}{ Pulmonary Contusion } & None & 0 \\
\hline & 1 lobe, unilateral & 1 \\
\hline & 1 lobe, bilateral & 2 \\
\hline & 2 lobes, unilateral & 2 \\
\hline & "<2 lobes, bilateral" (see below) & 3 \\
\hline & $\geq 2$ lobes, bilateral & 5 \\
\hline \multirow[t]{5}{*}{ Pleural involvement } & None & 0 \\
\hline & Pneumothorax & 1 \\
\hline & Unilateral Hemothorax or Hemopneumothorax & 2 \\
\hline & Bilateral Hemothorax or Hemopneumothorax & 3 \\
\hline & Tension Pneumothorax & 5 \\
\hline \multirow[t]{5}{*}{ Rib fractures } & 0 & 0 \\
\hline & 1 to 3 & 1 \\
\hline & 3 to 6 (will use 4 to 6 ), Unilateral & 2 \\
\hline & $>3$, Bilateral & 3 \\
\hline & Flail chest & 5 \\
\hline
\end{tabular}


Table 2-: Patient demographics

\begin{tabular}{|l|l|}
\hline Number of patients & 80 \\
\hline Mean age(mean \pm SDyears) & $40.12 \pm 13.75$ \\
\hline Sex of patient, n (\%) & $69(86.2)$ \\
Male & $11(13.8)$ \\
Female & \\
\hline Type of injury, n (\%) & $64(80)$ \\
Blunt & $16(20)$ \\
Penetrating & $48(60)$ \\
\hline Ventilated patients, n (\%) & \\
\hline thorax related complications,n (\%)-: & \multicolumn{2}{|l|}{} \\
\hline ARDS & $34(42.5)$ \\
\hline Pneumonia & $12(15)$ \\
\hline Second pneumonia & $8(10)$ \\
\hline BPF & $6(7.5)$ \\
\hline Empyema & $6(7.5)$ \\
\hline Mortality,n (\%) & $8(10)$ \\
\hline
\end{tabular}

Table-3: TTSS according to survival and ARDS

\begin{tabular}{|l|l|c|c|c|}
\hline \multicolumn{2}{|l|}{ Survival and ARDS } & TTSS X-ray & $\begin{array}{c}\text { TTSS Single } \\
\text { slice helical CT }\end{array}$ & $\begin{array}{c}\text { TTSS multislice(64 } \\
\text { row) CT / MDCT }\end{array}$ \\
\hline \multirow{2}{*}{ Survival } & Yes & $10.26 \pm 2.26$ & $14.00 \pm 3.02$ & $15.87 \pm 2.74$ \\
\cline { 2 - 5 } & No & $7.93 \pm 2.55$ & $9.81 \pm 3.36$ & $11.19 \pm 4.00$ \\
\hline p-value & & $0.005^{*}$ & $0.001^{*}$ & $0.002^{*}$ \\
\hline ARDS & Yes & $9.74 \pm 2.05$ & $12.82 \pm 2.77$ & $15.05 \pm 2.71$ \\
\cline { 2 - 5 } & No & $7.02 \pm 2.40$ & $8.32 \pm 2.76$ & $9.15 \pm 3.06$ \\
\hline p-value & & $0.0001^{1}$ & $0.0001^{*}$ & $0.0001^{*}$ \\
\hline
\end{tabular}

${ }^{1}$ Unpaired t-test

Fig.1: ROC analysis ofTTSS according to survival

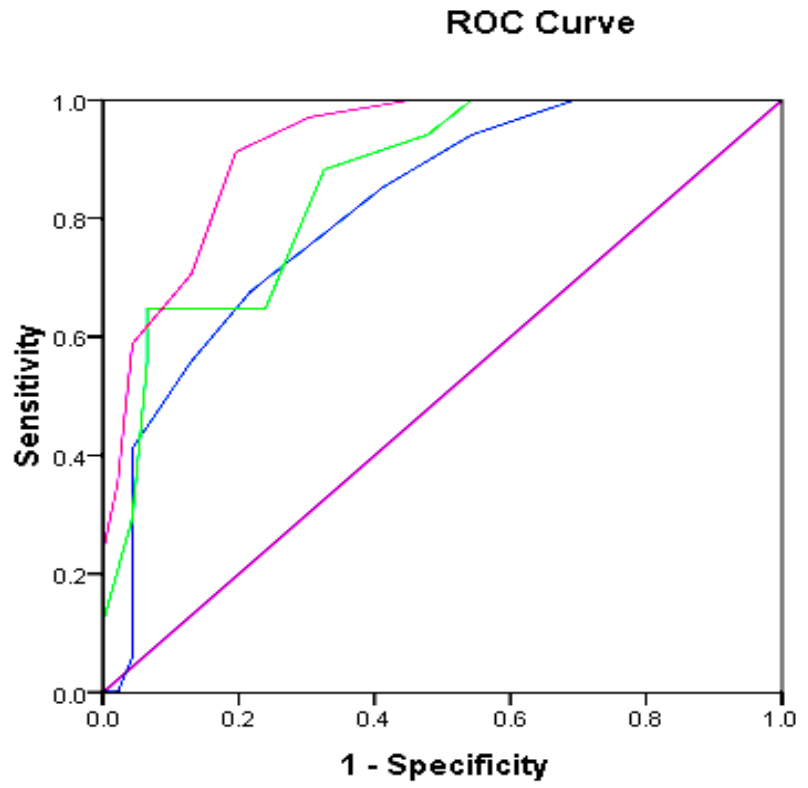

Source of the Curve

- TTSS -X-Ray

- TTSS- SSCT

- TTSS MDCT

- Reference Line 
Fig.-2: ROC analysis ofTTSS according to incidence of ARDS

RoC Curve

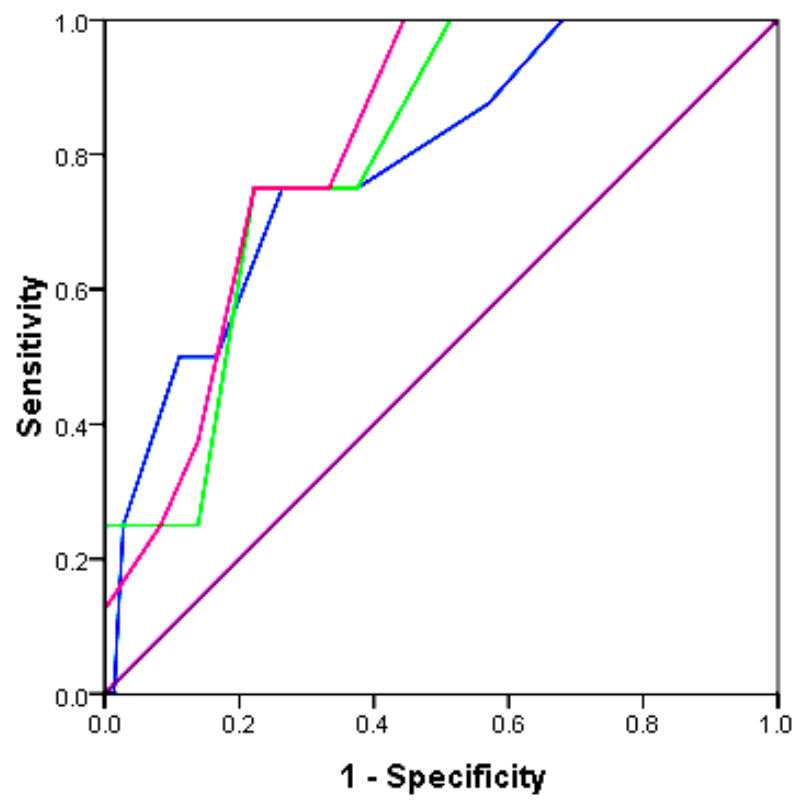

Diagonal segments are produced by ties.

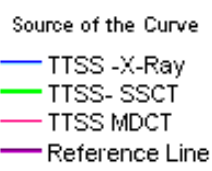

- Reference Line

\section{DISCUSSION}

This is the first study to validate the TTSS on the bases early ED CT findings as well as compared the TTSS calculated by radiological finding of conventional chest $\mathrm{x}$-ray and single slice helical CT for mortality and describe an association between the score and the development of ARDS. We used the results from the admission chest Xray to calculate TTSS, as originally described by Pape et al $2000{ }^{[7]}$ and also calculated TTSS by MDCT and single slice CT findings. When TTSS evaluated with radiological findings of MDCT, single slice CT along with conventional $\mathrm{x}$-ray, the score was highest 65 for MDCT in both group of patient (dead/alive and ARDS/non ARDS), with highly significant values. We did demonstrate a clear association between the TTSS and several outcome parameters. In addition our study was able to demonstrate an association between the TTSS and thorax-related death. The score was significantly higher than in patients who survived. We did not include patients who died of nonthorax related complication in our study cohort as per autopsy findings. This extra characteristic of the score could be of added value in trauma evaluation if we adopt the strategy "higher the value of TTSS, higher index of suspicion for fatal complications like ARDS or ICU care". We showed that the TTSS is significantly higher in patients who develop ARDS after thorax trauma. To our knowledge no other scoring system has demonstrated an association with the development of ARDS on the bases of early ED MDCT. This may lead to a different clinical policy on blood transfusion in patients with a high TTSS. Restrictive transfusion policies and ventilation strategies are advised for these patients because these measures are associated with a decreased incidence of ARDS ${ }^{[8]}$. As described by Wagner et al. if more than $28 \%$ of lung volume is affected patient need mechanical ventilation and intubation ${ }^{(4,8)}$. In concordance with trauma scores such as the New Injury Severity Score 152 (AUC of0.68) the TTSS demonstrated a high AUC of 0.81 which makes it a sensitive and specific scoring system for predicting mortality.AUC of TTSS was higher 
than AUC of New Injury Severity Score, indicating better score for predicting mortality in ED when early MDCT is carried out following emergency trauma protocol in stabilized patient. As the technical evolution of MDCT scanners, the limitations seen during the planning of the current studies have already been overcome. For instance, the reconstruction time of the whole set of wholebody images is no longer a major issue. However, the potential advantage of the most advanced MDCT technology has to be determined by means of prospective controlled trials. Further studies will be necessary to identify trauma patients who will benefit from whole-body MDCT screening in the early phase of multiple trauma care. Disadvantage of MDCT are high cost, availability only in tertiary medical centre. Radiation is more than the conventational CT but radiation risk for performing single study is less, a dose of 100 millisieverts $(\mathrm{mSv})$ is estimated to cause only 0.004 long-term mutations per cell. Sensitivity (70\% -98\%), specificity (95\%), false positivity $(5 \%-8 \%)$, false negativity $(10 \%)$ of MDCT ${ }^{(9)}$.

\section{CONCLUSION}

This study validate the Thoracic Trauma Severity Score (TTSS) with new emerging concept of early emergency Department Muti- row detector CT in secondary survey for predicting mortality in critically ill thoracic injury patients. As compared to TTSS x-ray study shows the significance of early MDCT in assessing volume of lung contusions and predicting ARDS and thereby need for mechanical ventilation in critically ill chest trauma patients. Thus shows the significance of early emergency MDCT in diagnostics and treatment decisions in traumatic lung injured patients.

\section{REFERENCES}

1. Vodicka J, Spidlen V, Safranek J, Simanek V, Altmann P. [Severe injury to the chest wall--experience with surgical therapy]. ZentralblChir. 2007;132 (6):542-6.
2. Pirente N, Blum C, Wortberg S, et al. Quality of life after multiple trauma: the effect of early onset psychotherapy on quality of life in trauma patients. Langenbecks Arch Surg. 2007;392 (6):739-745.

3. Vles WJ, Steyerberg EW, Essink-Bot ML, van Beeck EF, Meeuwis JD, Leenen LP. Prevalence and determinants of disabilities and return to work after major trauma.J Trauma. 2005;58(1):126-135.

4. Huber-Wagner S, Lefering R, Qvick LM, Korner M, Kay MV, Pfeifer KJ, Reiser M, Mutschler W, Kanz KG \& Working Group on Polytrauma of the German Trauma Society (2009) Effect of whole-body CT during trauma resuscitation on survival: a retrospective, multicentre study. Lancet 373(9673): 1455-146.

5. Salim A, Sangthong B, Martin M, Brown C, Plurad D \&Demetriades D (2006) Whole body imaging in blunt multisystem trauma patients without obvious signs of injury: results of a prospective study. Arch Surg 141(5): 468-473.

6. Nguyen D, Platon A, Shanmuganathan K, Mirvis SE, Becker CD \& Poletti PA (2009) Evaluation of a single-pass continuous whole-body 16-MDCT protocol for patients with polytrauma. AJR Am J Roentgenol 192(1): 3-10.

7. Pape HC, Remmers D, Rice J, Ebisch M, Krettek C, Tscherne H. Appraisal of early evaluation of blunt chest trauma: development of a standardized scoring system for initial clinical decision making. J Trauma. 2000;49(3):496-504.

8. Murray JF, Matthay MA, Luce JM, et al. An expanded definition of the adult respiratory distress syndrome. Am Rev Respir Dis 1988;138:720-3.

9. MowattG,Cumins E, Waugh N, Walker,MDCT An Evidence based Analysis,Ont health Techno Assess Ser. 2005;5(5):1-57. 


\section{Abbreviations}

BPF bronchopleural fistula

- CT computed tomography

- CXR chest radiograph

- ED CT emergency department computed tomography

- FAST focused assessment with sonography for trauma

- ICU intensive care unit

- ISS injury severity score

- MDCT multi-detector computed tomography

- PEEP positive end-expiratory pressure

- TTSS thorax trauma severity score

AIS abbreviated injury score• 